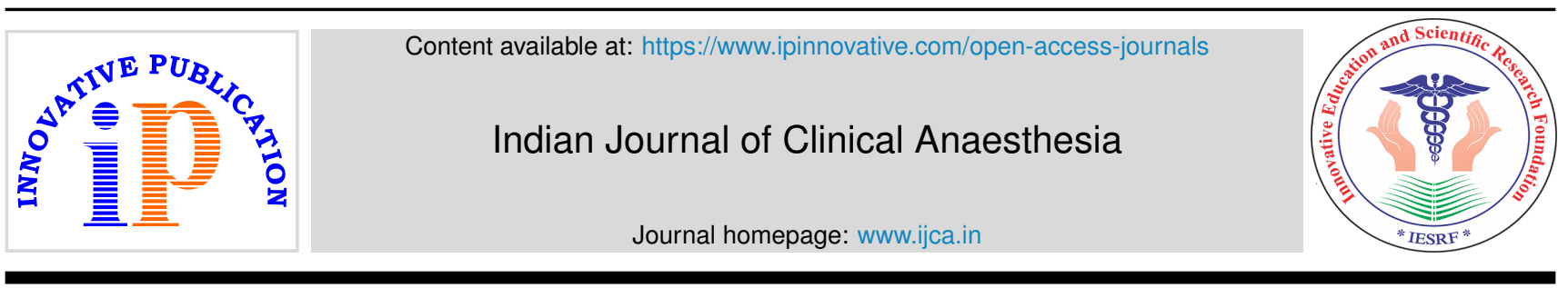

\title{
Editorial
}

\section{Evaluation of manuscripts- A guide to reviewers}

\author{
Pallavi Ahluwalia ${ }^{\circledR}{ }^{1, *}$, Bhavna Gupta ${ }^{2}$ \\ ${ }^{1}$ Dept. of Anaesthesia, Teerthanker Mahaveer Medical College, Moradabad, Uttar Pradesh, India \\ ${ }^{2}$ Dept. of Anaesthesia, All India Institute of Medical Sciences, Rishikesh, Uttarakhand, India
}

\section{A R T I C L E I N F O}

Article history:

Received 02-09-2020

Accepted 11-09-2020

Available online 15-03-2021

\begin{abstract}
A B S T R A C T
Peer-reviewed scientific publications are a source of high-quality and reliable information, especially valuable in today's world. In this guide to reviewers, I have tried to suggest how to scientifically read a paper and review it. It will be helpful for those who are starting to participate in peer review and I have summarised various questions related to the process. What are the points that should be considered when analysing the title, abstract, introduction, results, discussion and conclusion?

(C) This is an open access article distributed under the terms of the Creative Commons Attribution License (https://creativecommons.org/licenses/by/4.0/) which permits unrestricted use, distribution, and reproduction in any medium, provided the original author and source are credited.
\end{abstract}

\section{Introduction}

Peer review is a critical evaluation done by experts in the relevant field, of a scientific document such as a research article submitted for publication, a grant/study proposal/ protocol or a research program. ${ }^{1}$ During the initial reading, summarise what the manuscript is about in one or two paragraphs and its contribution to existing evidence. Note the document's strengths and weakness. Consider any issues that need to be corrected or fixed before publication. Initial paragraphs provide an overview and gives an idea that manuscript is reviewed thoroughly. They may also allow the editor, who might not be an expert in this specific area, to consider the study's more extensive meaning. Finally, these paragraphs would illustrate the manuscript's key messages that may be valuable for the readers. At first glance, read it once and reflect on the extensive background of the study. Reflect on-

What study question(s) are answered by the authors? Is there a strong case for why a topic is important? What the writers use approaches to address the research question? Whether still old methods are followed, when newer, more efficient approach is available? Are there any significant

\footnotetext{
* Corresponding author.

E-mail address: drpallaviahluwalia@yahoo.com (P. Ahluwalia).
}

flaws in the methodology? Are there any existing studies to improve the accuracy of the manuscript? If so, whether they are appropriate to make the research transparent? Will any additional evidence help to validate the conclusions presented and reinforce the paper?

Have the findings been adequately evaluated and interpreted? Does the data endorse the conclusions of the authors? Are the findings going to advance the area in a way? Will the value of the advance conform to the journal's standards? Would the manuscript work well together? Can it explicitly explain what has been achieved, why and what is the interpretation of findings? Was the manuscript wellstructured and easy to understand? If there are several inconsistencies in the manuscript, you may recommend that a fluent English speaker review the writers. If the text's content is obscure, you may suggest corrections before you examine it.

\section{Description, Abstract, and Keyword}

Title, abstract, and keywords can help future scholars locate the written paper. Abstracts must be a brief description of the entire manuscript and simple. It is crucial that the abstract is fascinating for the reader and ignites a spark to read the full article. Many users would read the summary than the whole article, but it should be more comfortable for 
readers to grasp without a technical context. Some of the concerns that need to be answered regarding the title of the manuscript, abstract, and principal terms would be:

1. Whether title tells what the research is all about and fully appropriate? If not, suggest another title?

2. Is manuscript effectively summarised in the abstract?

3. Is abstract simple and interpretable by a scholar not from of your specialty?

4. Does it provide enough information? Does the abstract include any unwanted details?

5. Any material mentioned in the abstract other than the manuscript's key text?

6. If recent, whether the keywords will allow the reader to locate an article? Do they describe and reflect the substance of the manuscript?

\subsection{Introduction}

The Introduction tells the reader about the manuscript. The Introduction provides the context to the study issue. When you analyze the Introduction, pose the following questions:

1. Is it enough to clarify the context so scholars outside your profession will appreciate it?

2. Can it correctly describe the current understanding of the study question?

3. Does the presentation include any needless information? Will this be made more concise?

4. Are the grounds for the analysis clear?

5. Are the goals of the analysis well established and compatible with the content of the manuscript?

\subsection{Materials and Methods}

The research methods are one of the most critical elements used to determine the paper's overall content. Also, this portion can provide readers with adequate knowledge to be willing to replicate the experiments. Reviewers should search for possible causes of prejudice in how the research was planned and performed and for areas where more clarification is required. The basic categories of knowledge in the Methodology segment can differ in various fields and research areas. However, basic rules for the Methodology pages are as follows:

1. The method of analysis should be explicitly defined. Whether informed consent was obtained; and what requirements or inclusion criteria's were followed for subjects to be included in the study. For e.g. whether ethical approval is there and CTRI registration done or not.

2. Whether the findings of the analysis and the outcome measurements are scientifically confirmed. Techniques employed to interpret the data should be scientifically accurate.
3. All materials and instruments, including the name and position of the source, should be listed. For, e.g., "Tests were performed with SPSS Software.

4. The Methodology segment should not include data that belongs to another section such as the Introduction or Results.

You may indicate that additional studies would significantly enhance the accuracy of the manuscript. Your recommendations should be compatible with the goals of the report. Note that more experiments may improve almost every thesis, so only recommend further work if you feel that the manuscript cannot be released without it.

\subsection{Results or Observations}

Readers would typically first glance at the cover, abstract, and findings of a manuscript. The results portion, including any statistics and graphs, are, therefore, several of the manuscript's most critical sections. You should closely review the estimates and tables to ensure that they correctly represent the findings. If you think it is essential, you might recommend improvements that will make the conclusions simpler to interpret.

1. Verify that the illustrated variables are well specified for the figures. Figures and tables should contain ambiguous metrics, such as standard error or confidence intervals and sample size.

2. Table headings and statistics should be adequately descriptive to comprehend the details without reading the main text comprehensively.

3. Search for instances where data is excessively replicated throughout figures, tables, or critical documents. The text should show the main observations or patterns, not copy data provided elsewhere. In the same manner, data to present a minimal amount of data may also be merged with another or omitted and substituted with a clarification about it in the text.

4. It is always appropriate to summarize the outcomes that are not essential to the analysis's goals rather than presenting them as results. However, non-display of relevant details or too much examples such as "data not shown" is inappropriate, and you should request that it be applied to the critical manuscript.

5. Check whether the data is displayed suitably; for example, identify whether the graph will offer better clarity than table? Do the statistics seem real and valid?

6. Check whether the interpretations are provided in the result section. This segment must include the actual findings and not the suggested ones. Whether Interpretations and inferences about findings are provided or not? 


\subsection{Statistics}

Most scientific manuscripts provide statistical analysis, and the thesis's outcomes rely on their findings. If the data is analysed or falsely recorded (fabrication), the document may strangle the readers. As a researcher and as a peer reviewer, one must have a sound understanding of statistics and closely analyse the statistical methodology and reporting of the manuscripts you are reading. If you don't thoroughly investigate the figures, notify the author about it in your feedback so that they know how to convince someone else to check them.

Such questions to be answered while analysing the methodological analyses and findings are as follows:

1. Is the scale of the sample acceptable and justified? Whether power analysis has been done during study design?

2. Check whether the results have fulfilled the assumptions of the tests used? Are the statistical tests used appropriately?

3. Have alternative causes of prejudice (e.g., confounding variables) been considered during analysis?

4. Wherever necessary, whether the p-values are reported? Ideally statistical comparisons in the text, statistics, and tables should be followed by a p-value. The statistical analysis portion could also establish the criterion for recognizing validity, such as 'P-value of 0.05 was found statistically important.'

\subsection{Discussions and Interpretation}

In the Discussion page, researchers can summarize the results, position them in the sense of previous studies, and clarify what they suggest for potential research, as well as for possible real-life implementations. If the author has not made these points as explicit as they should be, please mention this in your analysis. Some questions to be answered include:

1. Is the topic per the goals of the analysis set out in the Introduction?

2. Are there any alternative explanations of the results that the writers might have considered in their discussions?

3. Is there some general context to be identified in the Introduction segment other than the Discussion?

4. Have the investigators correctly correlated their results with the findings of other studies?

5. Are the researchers addressing the data in the Discussion? All related details should be discussed in the Results portion, while significant or noteworthy results can be outlined in the Discussion.

6. Have the authors mention any impact on future research?
7. Whether the shortcomings or limitations of the analysis identified? If not, what drawbacks did you find?

8. Are the findings of the authors backed by their data? Have the writers overestimated the significance of their results?

9. Pay attention to how the contributors use the citations when you scan through the content of the manuscript. Any problems that need to be checked include:

10. Are there places where the writers ought to quote a source and that they're not? In general, citations are required for all details but those that are well-established, widespread knowledge, or are specifically identified as the author's hypothesis. Do the investigators cite any of the most essential past research and clarify how they apply to current results? Notice which sources are lacking, if not. Are the studies cited recent enough to reflect new expertise on the subject?

11. Are the writers referring to the study of several study groups? In particular, it is preferable to quote papers from one or two research groups, mainly if one of the most cited groups is an authors (although it is not always possible in very small fields of study). Do the writers cite a variety of research articles? Are they recent?

\section{Analysis of Review Papers}

A review paper is published to summarize the existing state of knowledge on the topic. Peer review on these kinds of papers involves a somewhat different set of standards relative to empiric articles. If a systematic review/metaanalysis approach is employed, it is not relevant or published. The review article's content can be measured on factors such as timeliness, depth, and consistency of the topic and whether it points forth the best directions for potential study. The study article should include an authoritative overview of the existing knowledge of the subject. Thus, the peer reviewer must always analyse the studies selected in the report. The composition and flow is also significant in an analysis article as it includes a vast volume of explicit material.

\subsection{Reading the reviewer's paper}

If you are endorsing or refusing a paper, remember that your aim is to support writer to improve this and their future manuscript. Avoid unnecessarily critical language or personal remarks, point out the novel's key benefits and shortcomings, and recommend concrete approaches to solve the issues you find. Also, avoid making concise and blunt statements. Most publications are reviewed anonymously, so avoid remarks that clarify your name to the writers. You can compose the report in the requested style, if the journal 
has provided detailed guidelines for the reviewer of the article, or a template is to be filled as part of the analysis. If there is unclear guidance, the summary can be divided into two parts:

1. Remarks for the editor

2. Inputs to be reviewed by both editors and authors

\subsection{Remarks for the editor only}

This includes your suggestion for the manuscript and the reasons behind it. This should focus on empirical soundness, novelty, consistency, significance, and suitability of the manuscript for publication. Editors take several things into account when determining if a paper is right for their journal, so it is beneficial to include justification or argument for the recommendation. Guidelines are typically those of the following: approve the document in its original state, publish with slight modifications, and publish only if significant revisions have been produced, or refuse the article.

\subsection{Comments on all editors and authors}

Write a thorough report on the various sections of the manuscript in this portion. Start with a brief summary of the manuscript, which you wrote initially. Then, clarify the problems you have defined and that need to be resolved preferably in a list. Categorise into main issues and minor issues. Then suggest key points, like difficulties with the research process or review. First, write regarding small problems that could involve difficult-to-read tables or numbers, sections that require further detail, and ideas for removing redundant content. If you believe that the English language of the text is not appropriate for print, strive to provide clear explanations so that the writers know when and how to cope with the issues. Precisely mention the shortcomings of the manuscript and how to fix them. Provide the page and the line number(s) (if the manuscript contains a line number) relevant to the analysis section that you are addressing. This would enable editors to determine if the authors have resolved their updated document issues at a later stage. Remember that all the contributors may not be fluent English speakers. After you finish writing, always read your comments to ensure if you have used consistent, straightforward language and that the explanations for your suggested improvements are evident. Good science is gender blind, colour blind and age blind. ${ }^{2}$

\subsection{Following the analysis}

Once you send a review, you should get a notice that the review has been successfully uploaded.

1. Certain journals may notify reviewers whether the manuscript has been approved or denied, while others may not.

2. Certain publications submit feedback from other reviewers to the same manuscript along with a judgment letter; reading these comments will help you enhance your potential ratings. If the writers update and resubmit the manuscript after revision, the editor may also review the edits and determine if the reviewer's suggestions have been thoroughly answered. If anything occurs, concentrate on whether the writers have addressed the issues you found in the first analysis.

To summarise, peer review helps us build a strong foundation for scientific research. Reviewer gets the opportunity to learn something new, improve the quality of published work and contributes to advancements in his or her own field.

\section{Conflict of Interest}

None.

\section{References}

1. Gallin J, Ognibene F, Johnson LL. Principles and Practice of Clinical Research (Fourth Edition); 2018.

2. Mahmoudi M. A Healthier Peer Review Process Would Improve Diversity. ACS Appl Mater Interfaces;2020(37):40987-40989.

\section{Author biography}

Pallavi Ahluwalia, Professor (ㄷ https://orcid.org/0000-0001-8730-3730

Bhavna Gupta, Assistant Professor

Cite this article: Ahluwalia P, Gupta B. Evaluation of manuscripts- A guide to reviewers. Indian J Clin Anaesth 2021;8(1):1-4. 